\title{
Alternative Splicing of Three Genes Encoding Mechanotransduction-Complex Proteins in Auditory Hair Cells
}

\author{
Zijing Zhou, ${ }^{1}$ Xiaojie Yu, ${ }^{1}$ Biaobin Jiang, ${ }^{1,2}$ Wanying Feng, ${ }^{6}$ Ye Tian, ${ }^{1}{ }^{1}$ Zhiyong Liu, ${ }^{7}$ Jiguang Wang, ${ }^{1,2,3}$ \\ and Pingbo Huang ${ }^{1,2,3,4,5}$
}

https://doi.org/10.1523/ENEURO.0381-20.2020

${ }^{1}$ Division of Life Science, Hong Kong University of Science and Technology, Hong Kong, China, ${ }^{2}$ Department of Chemical and Biological Engineering, Hong Kong University of Science and Technology, Hong Kong, China, ${ }^{3}$ State Key Laboratory of Molecular Neuroscience, Hong Kong University of Science and Technology, Hong Kong, China, ${ }^{4}$ HKUST Shenzhen Research Institute, Hong Kong University of Science and Technology, Hong Kong, China, ${ }^{5} \mathrm{Hong}$ Kong Branch of Guangdong Southern Marine Science and Engineering Laboratory (Guangzhou), Hong Kong University of Science and Technology, Hong Kong, China, ${ }^{6}$ Bioscience and Biomedical Engineering Thrust, The Hong Kong University of Science and Technology (Guangzhou), Hong Kong, China, and ${ }^{7}$ Institute of Neuroscience, Chinese Academy of Science, Shanghai, China

\section{Visual Abstract}
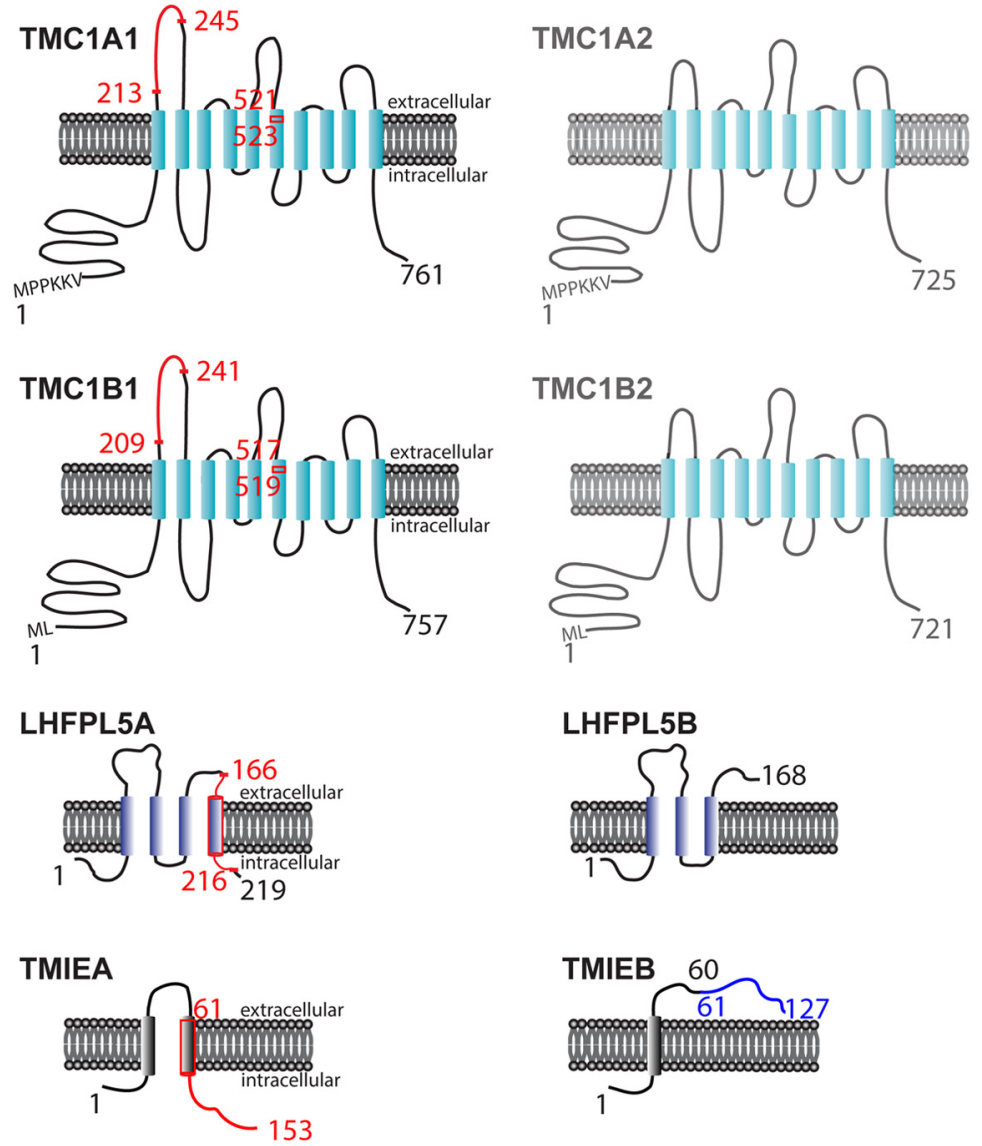


\section{Significance Statement}

We have identified previously unreported splicing variants of transmembrane channel-like protein 1 (TMC1), lipoma HMGIC fusion partner-like 5 (LHFPL5), and transmembrane inner ear protein (TMIE), the pivotal molecules forming the hair-cell mechanotransduction (MT) machinery in the inner ear. Our findings reveal the potential complexity of the MT-complex composition and provide valuable guidance for future research on the function, regulation, and trafficking of TMC1, LHFPL5, and TMIE. Furthermore, our study could help direct the clinical diagnosis of hearing loss related to aberrant splicing of TMC1, LHFPL5, and TMIE.

The mechanotransduction (MT) complex in auditory hair cells converts the mechanical stimulation of sound waves into neural signals. Recently, the MT complex has been suggested to contain at least four distinct integral membrane proteins: protocadherin 15 (PCDH15), transmembrane channel-like protein 1 (TMC1), lipoma HMGIC fusion partner-like 5 (LHFPL5), and transmembrane inner ear protein (TMIE). However, the composition, function, and regulation of the MT-complex proteins remain incompletely investigated. Here, we report previously undescribed splicing isoforms of TMC1, LHFPL5, and TMIE. We identified four alternative splicing events for the genes encoding these three proteins by analyzing RNA-seq libraries of auditory hair cells from adult mice [over postnatal day (P)28], and we then verified the alternative splicing events by using RT-PCR and Sanger sequencing. Moreover, we examined the tissue-specific distribution, developmental expression patterns, and tonotopic gradient of the splicing isoforms by performing semiquantitative and quantitative realtime PCR (qRT-PCR), and we found that the alternative splicing of TMC1 and LHFPL5 is cochlear-specific and occurs in both neonatal and adult mouse cochleae. Our findings not only reveal the potential complexity of the MT-complex composition, but also provide critical insights for guiding future research on the function, regulation, and trafficking of TMC1, LHFPL5, and TMIE and on the clinical diagnosis of hearing loss related to aberrant splicing of these three key genes in hearing.

Key words: alternative splicing; auditory hair cell; ion channel; mechanosensation; tonotopic gradient

\section{Introduction}

The mechanotransduction (MT) complex is a channelcontaining macromolecular complex in auditory hair cells that converts the mechanical stimulation of sound waves into neural signals in auditory perception. The precise molecular composition of this macromolecular transducer remains incompletely established, but the MT complex has been suggested to contain at least four membrane proteins: transmembrane channel-like protein 1 (TMC1), protocadherin 15 (PCDH15), lipoma

Received August 31, 2020; accepted December 10, 2020; First published January 28, 2021.

The authors declare no competing financial interests.

Author contributions: Z.Z., B.J., Z.L., J.W., and P.H. designed research; Z.Z., X.Y., W.F., and Y.T. performed research; Z.Z. and P.H. analyzed data; Z.Z. and P.H. wrote the paper.

We thank Prof. Tuan Anh Nguyen's group for the suggestion of using the strategy of primer-template mismatch. This work was supported by Hong Kong RGC Grants GRF16102417, GRF16100218, and GRF16102720, the National Natural Science Foundation of China-RGC: Research Grants Council Joint-Research Scheme Grant N_HKUST614/18, the Shenzhen Basic Research Scheme Grant JCYJ20170818114328332, the Southern Marine Science and Engineering Guangdong Laboratory (Guangzhou) Grant SMSEGL20SC01-K, and in part by the Innovation and Technology Commission Grant ITCPD/17-9.

Correspondence should be addressed to Pingbo Huang at bohuangp@ ust.hk.

https://doi.org/10.1523/ENEURO.0381-20.2020

Copyright @ 2021 Zhou et al.

This is an open-access article distributed under the terms of the Creative Commons Attribution 4.0 International license, which permits unrestricted use, distribution and reproduction in any medium provided that the original work is properly attributed.
HMGIC fusion partner-like 5 (LHFPL5), and transmembrane inner ear protein (TMIE). The human orthologs of TMC1 and TMIE were first identified as products of genes associated with nonsyndromic hearing loss (Kurima et al., 2002; Naz et al., 2002), and LHFPL5 was first reported as the protein whose mutation caused deafness in hurry-scurry mice (Longo-Guess et al., 2005). TMC1 and TMIE were recently identified as components of the MT channel (Kawashima et al., 2011; Kim and Fettiplace, 2013; Pan et al., 2013, 2018; Cunningham et al., 2020), PCDH15 forms part of the tip link that is generally accepted to serve as the gating spring of the MT channel (Ahmed et al., 2006; Bartsch et al., 2019), and LHFPL5 is considered to functionally couple the tip link to the MT channel and stabilize TMC1 expression (Xiong et al., 2012; Beurg et al., 2015; Yu et al., 2020). Moreover, these MT-channel components have been found to display specific spatiotemporal patterns of expression. For example, TMC1 mRNA is specifically expressed in the cochlea (and several other tissues; Keresztes et al., 2003) and also exhibits a specific temporal pattern, it is first detected at postnatal day $(P) 4$ and is maintained through adulthood in the cochlea (Kawashima et al., 2011); moreover, TMC1 protein expression gradually increases along the tonotopic axis from the apex to the base in outer hair cells (OHCs; Beurg et al., 2018). Despite these recent notable advances in our understanding of the MT complex, the composition, function, and regulation of the MT-complex proteins remain to be comprehensively investigated.

Alternative splicing, which occurs in $~ 95 \%$ of multiexonic genes in humans (Pan et al., 2008), substantially 
increases the diversity of the proteins that can be encoded by the genome, and this protein diversity contributes to tissue-identity acquisition, organ development, and tissue and organ physiology (Baralle and Giudice, 2017; Liu et al., 2017). For example, numerous splicing variants of the mechanosensitive channel PIEZO2 have been found to be expressed in a cell-type-specific manner in sensory ganglia, and these isoforms confer distinct biophysical properties that allow the detection of different types of mechanical stimuli (Szczot et al., 2017). Furthermore, in the case of the mechanosensitive twopore-domain potassium channel TREK-1, a splicing isoform resulting from an alternative stop codon in the retained intron, TREK-1e, has been identified, and coexpression of TREK-1e with TREK-1 reduces the surface expression of TREK-1 in HEK293T cells (Rinné et al., 2014), this finding indicates that alternative splicing isoforms can regulate the trafficking of the major isoform of a protein. The advent of next-generation sequencing techniques and the development of various computational tools have substantially enhanced our ability to identify alternative splicing at the genome-wide level.

To comprehensively understand the function and regulation of the MT complex, it is crucial to investigate the precise composition of the MT complex, regardless of whether the complex contains other unknown proteins or distinct splicing variants of the known components. Previous studies have identified three splicing variants of PCDH15 (Pepermans et al., 2014) and two splicing variants, featuring alternative translation start sites, of TMC1 (Kawashima et al., 2011). Here, by using publicly available data from mouse auditory hair cells (Li et al., 2018), we identified previously unreported splicing variants of TMC1, LHFPL5, and TMIE, and we then performed RTPCR and Sanger sequencing to verify the alternative splicing events. The complexity and accuracy of the MTcomplex composition revealed by our findings should be carefully considered in future research and in clinical diagnosis of deafness.

\section{Materials and Methods}

\section{Bioinformatics}

Illumina reads of a recently published cDNA-seq library of auditory hair cells of adult mice (Li et al., 2018) were aligned to $\mathrm{GRCm} 38$ ( $\mathrm{mm} 10)$ available from Ensembl by using the rapid and sensitive alignment program HISAT2 (Pertea et al., 2016). The Mixture of Isoforms (MISO) Sashimi Plot Feature [Integrative Genomic Viewer (IGV); Broad Institute] was used to visualize alternative splicing events.

\section{Mice}

C57BL/6 background mice were used for experiments. All animal procedures were approved by the University Committee on Research Practices at the Hong Kong University of Science and Technology (the ethics research project number A19005).

\section{RNA extraction}

For use in conventional PCR studies, we dissected the cochlea from four P8 mice of either sex and the cerebrum, cerebellum, cochlea, colon, eye, and testis from three adult (P32-P40) male mice. For quantitative real-time PCR (qRT-PCR), the organ of Corti was dissected from 15 P6 mice of either sex. The distal end at the basal side of the tissues, which was frequently damaged during dissection, was discarded to ensure the integrity of four rows of auditory hair cells, and the remaining part was divided equally into apical, middle, and basal segments (Fig. $3 C$ ). The same segments from three mice were pooled for one data point. Tissues were homogenized in an RNase-free glass abrader and total RNA was extracted by using a Minibest universal RNA extraction kit (Takara) according to the manufacturer's protocol. RNA concentration and purity were determined using a Nanodrop 2000 spectrophotometer (Thermo Fisher Scientific).

\section{PCR and validation of isoforms}

To validate the existence of the splicing isoforms of Tmc1, Lhfp/5, and Tmie, primers were designed to target exon 8 and exon 10 of Tmc1, exon 1/2 and exon 3 of Lhfp/5, or exon 4 and exon 5 of Tmie flanking the alternative splicing region; two pairs of unique primers were also designed for verifying the alternative splicing event in exon 14 of Tmc1 (see details in the legend for Fig. 2B). Total RNA was reverse-transcribed into cDNA by using a High-Capacity RNA-to-cDNA kit (Thermo Fisher Scientific). The amount of cDNA used as the PCR template was equivalent to $30 \mathrm{ng}$ of total RNA.

PCR was performed using KAPA HIFI polymerase (Kapa Biosystem). To amplify the alternative splicing isoforms for Sanger sequencing, we used two rounds of $\mathrm{PCR}$, each with 32 cycles of reaction, and an annealing temperature of $60^{\circ} \mathrm{C}$. The PCR products from the first round of PCR were separated in $2 \%$ agarose gels, and the bands that were of the size predicted for the alternative splicing isoforms were excised and purified for use as the template for the second round of PCR. The final amplified PCR products were purified and subject to Sanger sequencing.

For semiquantitative PCR, the process and conditions were identical to those mentioned above, but only one round of PCR was used and the PCR products were then electrophoretically separated and visualized using a ChemiDoc MP Image System (Bio-Rad).

The primers used in these experiments are listed in Table 1.

\section{qRT-PCR}

To separate the signal of the alternative splicing isoform from that of the constitutive splicing isoform, reverse primers harboring isoform-specific sequences were used. Total RNA was reverse-transcribed into cDNA by using a high-capacity RNA-to-cDNA kit, and the amount of cDNA used as the template for each reaction in qRT-PCR was equivalent to $4.6 \mathrm{ng}$ of total RNA. The primers used are listed in Table 2. 
Table 1: Primers for conventional PCR (including nested PCR and semiquantitative PCR)

\begin{tabular}{ll}
\hline Primer name & Sequence \\
\hline Tmc1 exon 8 F & AGTGGCCTCGTACTTCCTGTT \\
Tmc1 exon 10 R & CGTGCGTTTATTGTCGTAATAGCC \\
Tmc1 exon 14 F_1 & TCGTTCATCCTGCAGATG \\
(primer 1 in Fig. 2B) & \\
$\begin{array}{c}\text { Tmc1 exon 15 R } \\
\text { (primer 2 in Fig. 2B) }\end{array}$ & ACGTAAGTGGTCAGGACG \\
Tmc1 exon 14 F_1' & CACAGGAGCACCCTTाT \\
(primer 1' in Fig. 2B) & \\
Tmc1- 19 bp-span R & TGAGACGCACGAATTCCA \\
(primer 2' in Fig. 2B) & \\
Lhfp/5 exon 1 F_1 & CCATCATCTGCTTCAGCCTG \\
Lhfp/5 exon 3 R_1 & ACCTCGGTTGCTTCAGACTT \\
Lhfp/5 exon 1 F_2 & GCCTTCAAGACTGCCATGTTC \\
Lhfp/5 exon 3 R_2 & GAATTGTTGCTGCAGCACC \\
Tmie exon 4 F & ACCAAGGAGACTGTGGTGTT \\
Tmie exon 5 R & AGCCTCGATCTCCTTCCGC \\
\hline
\end{tabular}

$F$, forward primer; $R$, reverse primer.

Reaction mixtures were prepared using LightCycler 480 SYBR Green I Master Mix (Roche), as per the manufacturer's protocol, and qRT-PCR was performed using a LightCycler 480 instrument (Roche). The $\mathrm{C}_{\mathrm{T}}$ value was determined using a built-in fit-points method. The ratio of concentration of each gene relative to GAPDH gene was calculated using the $\Delta \Delta \mathrm{C}_{\mathrm{T}}$ method.

\section{Statistics}

All data are shown as mean \pm SEM. Statistical analysis was performed using Student's $t$ test, and $p<0.05$ was considered statistically significant.

\section{Results}

\section{In silico analysis of alternative splicing in Tmc1, Lhfpl5, and Tmie}

Owing to the development of second-generation sequencing techniques, RNA-seq data of rare cells such as auditory hair cells have now become available. By using a recently published cDNA-seq library of auditory hair cells of adult mice (Li et al., 2018), we analyzed alternative splicing in the genes Tmc1, Lhfp/5, and Tmie (see Materials and Methods) and obtained the following results.

First, we found that Tmc1 starts at exon 1 (and then skips exon 2) or alternatively starts at exon 2 (data not

Table 2: Primers for qRT-PCR

\begin{tabular}{ll}
\hline Primer name & Sequence \\
\hline mGAPDH $\mathrm{F}$ & TCACCACCATGGAGAAGGC \\
mGAPDH R & GCTAAGCAGTTGGTGGTGCA \\
Tmc1 $\mathrm{F}$ & GTCAGTTGGTTCCTCAGTGGC \\
Tmc1 constitutive R & CTAGGTAAGCTGCCGTACGG \\
Tmc1 alternative R & GCCAGGCCCTCCGGTAA \\
Lhfpl5 F & CCATCATCTGCTTCAGCCTG \\
Lhfp/5 constitutive R & CATGAAGGCCCAGCGGAT \\
Lhfpl5 alternative R & GACTTCCTCCGTCTGCTCG \\
\hline
\end{tabular}

$\mathrm{F}$, forward primer; $\mathrm{R}$, reverse primer. shown) as previously reported (Kawashima et al., 2011), the two isoforms are designated as TMC1A (starting at exon 1) and TMC1B (starting at exon 2). TMC1A is considerably more abundant than TMC1B (ratio 95:5) and is therefore considered to be the canonical form (Yamaguchi et al., 2020). More importantly, we identified two previously undescribed alternative splicing events: exon 9 skipping and alternative $3^{\prime}$ splicing in exon 14 at chr19:20 823987 (Figs. 1A, 4).

Second, we identified an alternative $3^{\prime}$ splicing site in exon 2 of Lhfp/5 at chr17:28580014 (Fig. 1A). Intriguingly, this alternative splicing site was fortuitously detected and suggested as a cryptic splicing site in Lhfp/5 $5^{\text {hscy-2J }}$ mice, in which the normal splicing donor-recognition site of exon 2 was disrupted as a result of $\mathrm{N}$-ethyl-N-nitrosourea-induced mutagenesis and hearing was severely damaged (Longo-Guess et al., 2007). We designated the fulllength, constitutive splicing LHFPL5 isoform as LHFPL5A and the alternative splicing isoform as LHFPL5B (Fig. 4). LHFPL5A is substantially more abundant than LHFPL5B (Fig. $1 A$, ratio $\sim 93: 7$ ) and is thus regarded as the canonical form.

Third, we also identified an alternative $3^{\prime}$ splicing site in Tmie exon 4 at chr9:110870665 (Fig. 1A). The constitutive and alternative splicing isoforms of TMIE (ratio 95:5) were designated as TMIEA and TMIEB, respectively (Fig. 4).

All the newly identified splicing events showed the highest junction coverage and could be reproduced in at least four out of the five batches of biological replicates in the study of Li et al. (2018; Fig. 1A). Interestingly, both of the newly identified alternative splicing events in TMC1 and the alternative splicing event in TMIE appeared to feature a higher ratio in $\mathrm{OHCs}$ than in inner hair cells (IHCs; Fig. $1 B)$. The alternative splicing ratio was determined by dividing the number of alternative splicing junctions by the total number of junctions (alternative and constitutive). By contrast, the alternative splicing ratio of LHFPL5 was similar in OHCs and IHCs (Fig. 1B). Because RNA-seq data for human inner ear are unavailable, we analyzed the conservation of the splicing donor/receptor sites and the nearby adjunct nucleotides between mice and humans. The high conservation observed indicates that the alternative splicing events might occur in humans as well (Fig. 1C).

One concern regarding RNA-seq is that the reversetranscription process in this technique might disproportionally amplify shorter isoforms because the dT primer targets the poly-A region and longer isoforms are more difficult to reverse-transcribe than shorter isoforms; this could lead to overestimation of the ratio of alternative/constitutive splicing isoforms. A similar concern could apply to PCR amplification of cDNA. However, the length differences between the transcripts of the alternatively and constitutively spliced isoforms in this study were small (length ratios: TMC1B2/TMC1B1 = 0.973; LHFPL5B/LHFPL5A = 0.88; TMIEB/TMIEA = 0.988) and presumably would have minimally affected the analysis of the ratio of alternative/ constitutive splicing isoforms. 
A

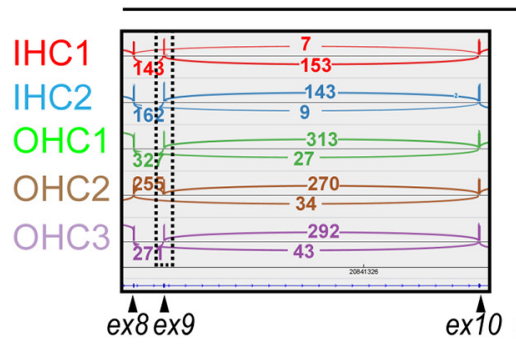

Constitutive

Alternative

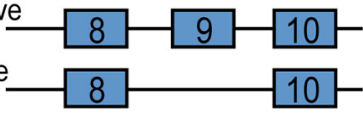

B
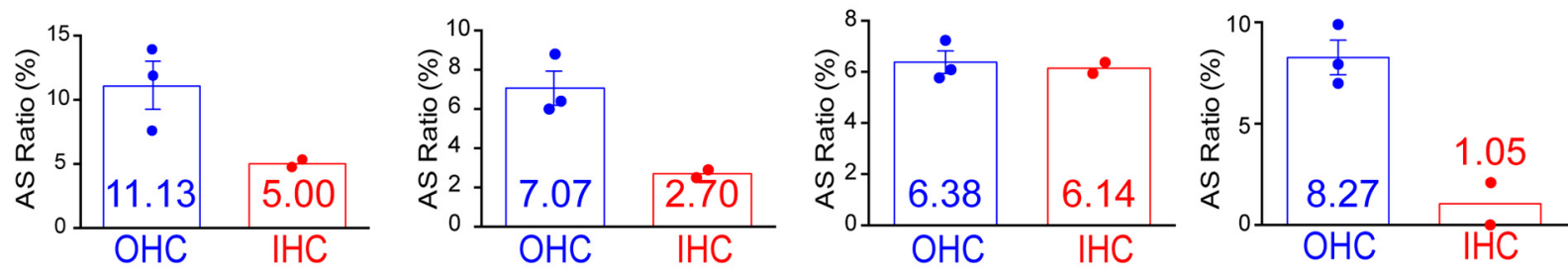

C
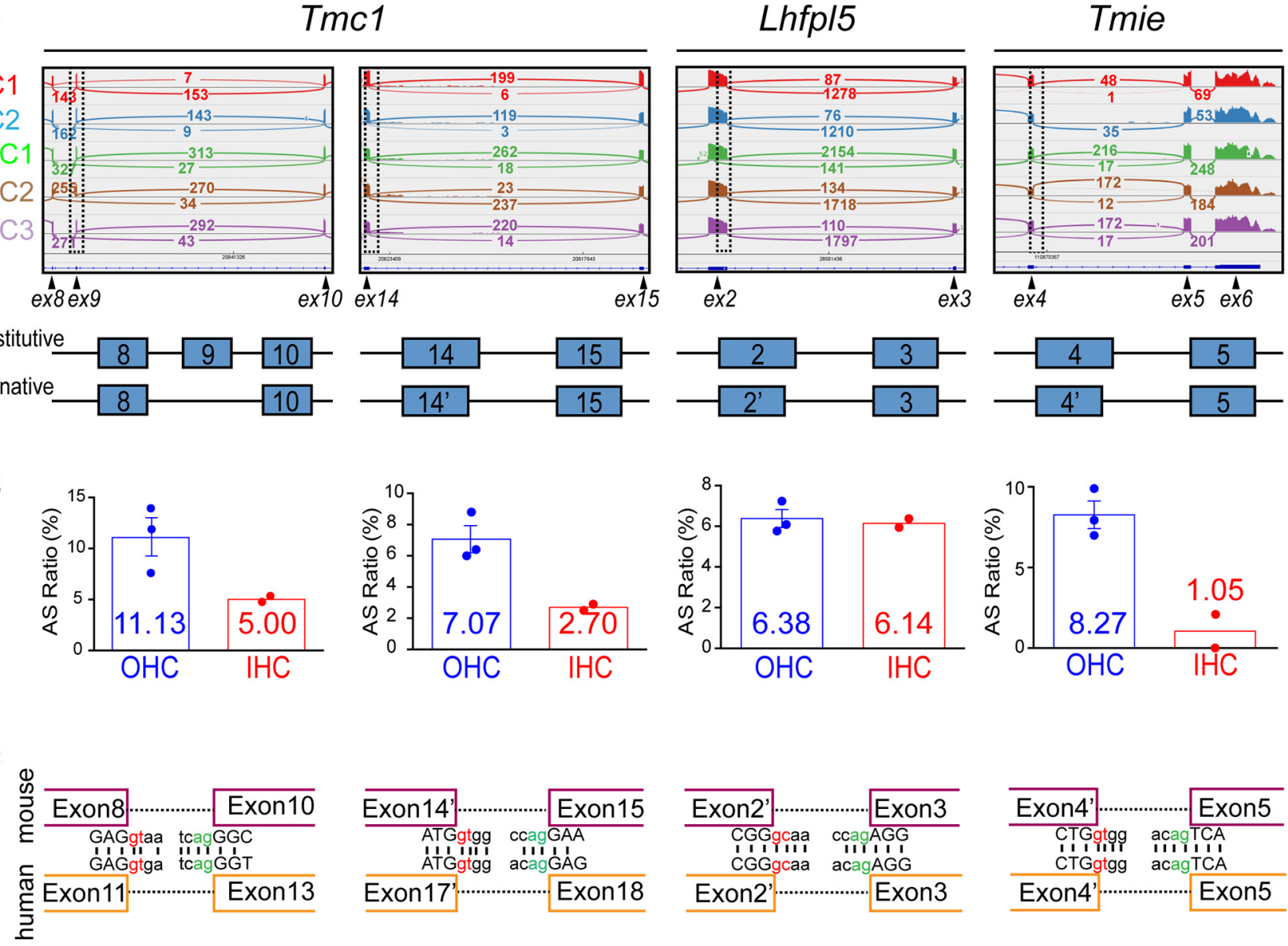

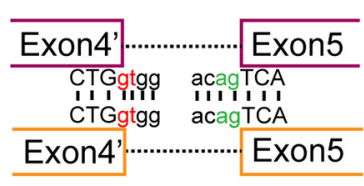

Figure 1. In silico analysis of alternative splicing in Tmc1, Lhfp/5, and Tmie. A, upper panel, Sashimi plots showing coverage of exons and junctions. Individual biological replicates are indicated using different colors; rectangular boxes: regions where alternative splicing events occur. Exons (ex) corresponding to peaks of reads are indicated below. Lower panel, Schematic of constitutive and alternative splicing isoforms; lines: introns. $\boldsymbol{B}$, Corresponding ratios of alternative splicing (AS) events to total splicing (constitutive and alternative) events illustrated in panel $\boldsymbol{A}$. AS ratios in IHCs and OHCs are shown; AS ratio = number of junctions using alternative splicing sites/number of junctions using both constitutive and alternative splicing sites. $\boldsymbol{C}$, Conservation in mice and humans of the corresponding splicing sites for the four alternative splicing events depicted in panel $\boldsymbol{A}$. Exons upstream and downstream of splicing sites are represented in purple (mouse) or orange (human). Donor sites (gc for splicing event in Lhfp/5 and gt for others) are shown in red, acceptor sites (ag) in green. Adjunct sequences in exons are uppercased, intronic sequences are lowercased. Vertical bars, identical nucleotides.

\section{Validation of splicing events in Tmc1, Lhfp/5, and Tmie through Sanger sequencing}

To eliminate potential artifacts in in silico analyses, alternative splicing identified by analyzing RNA-seq data must be verified using Sanger sequencing. Here, sequencing results obtained using primers flanking the predicted alternative splicing sites in Tmc1 (exon 9 skipping; Fig. 2A), Lhfp/5 (Fig. 2C), and Tmie (Fig. 2D) confirmed the existence of three splicing events in Tmc1 (exon 9 skipping), Lhfp/5, and Tmie. However, this approach involving the use of primers flanking the predicted alternative splicing site cannot be used to verify the alternative splicing event in exon 14 of Tmc1; this is because the alternative splicing removes only nine nucleotides (Figs. 1A, 2B), and the alternative splicing isoform therefore cannot be resolved without contamination by the predominant constitutive splicing isoform in the agarose-gel electrophoresis performed before Sanger sequencing, although the PCR products were as short as $\sim 100 \mathrm{bp}$ and showed clear separation (Fig. $2 B I, I I)$. Thus, as an alternative, we used the strategy of primer-template mismatch (Kwok et al., 1990; Stadhouders et al., 2010).

In principle, every mismatch between a primer and template reduces priming efficiency and overall PCR product yield; however, mismatches in the last five bases of the $3^{\prime}$-end region of a primer produce a considerably stronger effect than do mismatches located more toward the 5' end (Kwok et al., 1990; Stadhouders et al., 2010). Intriguingly, this undesirable effect of primer-template mismatch can be useful in basic research and molecular diagnostics: it enables differential detection of nucleic acid sequences featuring small differences, such as in the 
A

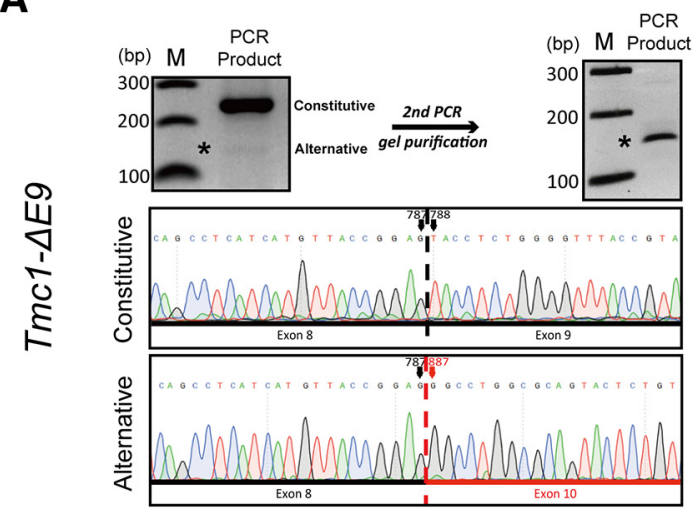

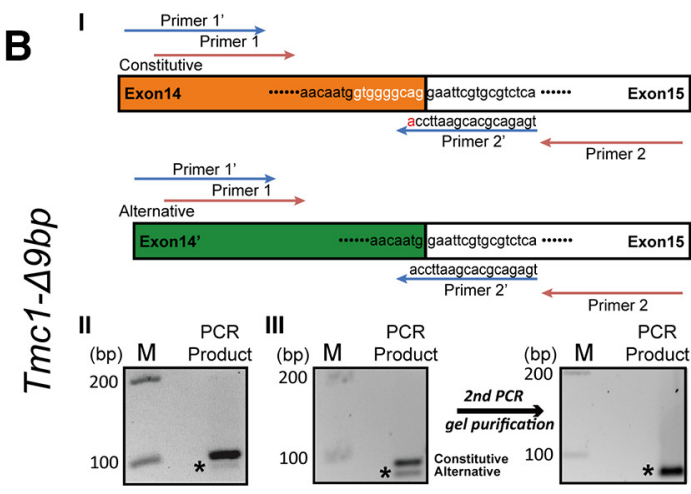

IV

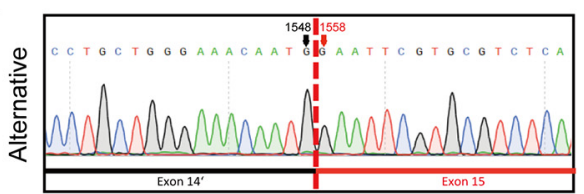

C

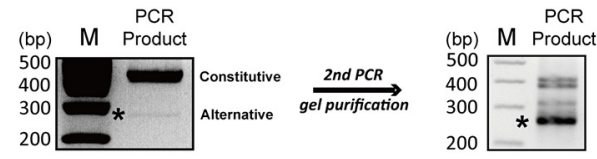

$\frac{\log }{5}$
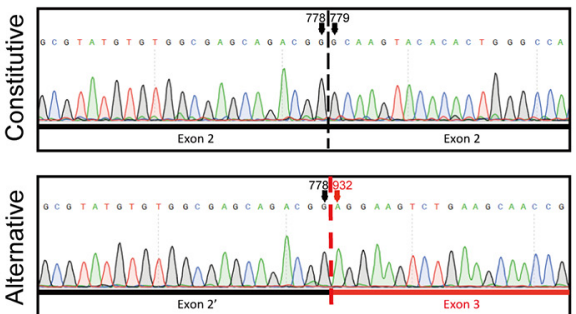

D

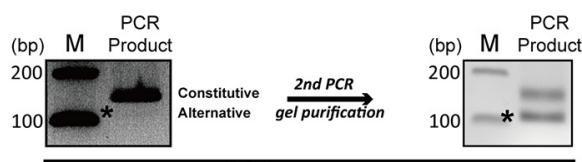

$\stackrel{0}{\stackrel{0}{\Sigma}}$
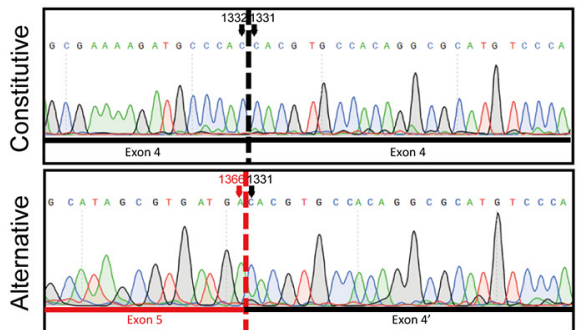

Figure 2. Validation of splicing events through Sanger sequencing. A, Mouse cochlear cDNA was prepared through reverse transcription and used in the first round of PCR with primers flanking predicted splicing sites in Tmc1 (exon 9 skipping). After PCR products were separated using agarose-gel electrophoresis (left, upper panel), the band at the predicted size for the constitutive splicing variant was excised, purified, and subject to Sanger sequencing. The band at the predicted size for alternative splicing variants was excised, purified, and used as the template for the second round of PCR (right, upper panel); the final PCR products in the second round were purified and subject to Sanger sequencing. * , alternative splicing isoforms. Electropherograms are presented in middle and lower panels; numbers at the top of electropherograms indicate nucleotide positions in the mRNA sequence annotated by NCBI. B, Validation of Tmc1 splicing: 9-bp skipping at 3' end of exon 14. Mouse cochlear cDNA was prepared through reverse transcription and used as the template for the PCR in panel II and for the first round of PCR in panel III; the band at the predicted size for the alternative splicing variant in the first round of PCR in panel III was excised, purified, and used as the template for the second round of PCR. Primers 1/2 (orange) and 1'/2' (blue) were used for PCR in panels II, III, respectively; primer 2' contained one base mismatched to the constitutive splicing isoform (panel $\boldsymbol{I}$ ). The PCR product of the second round of PCR in panel III was inadequately long for clear sequencing of the region around the alternative splicing site, and therefore the product was slightly extended by cloning it into pcDNA3 plasmid before Sanger sequencing. $\boldsymbol{C}, \boldsymbol{D}$, Experiments similar to $\boldsymbol{A}$ performed for $L$ hfp/5 (C) and Tmie $(\boldsymbol{D})$. P8 mice were used in all experiments; $\mathrm{M}$, molecular-weight size markers.

detection of single nucleotide polymorphisms and in allele-specific PCR (Stadhouders et al., 2010). Here, we designed a special reverse primer for Tmc1 (Figure 2BI, primer $2^{\prime}$ ) that crosses over the alternative splicing site by two nucleotides and therefore features a one-nucleotide mismatch with the constitutive splicing isoform while perfectly matching the alternative splicing isoform; notably, the one-nucleotide mismatch drastically reduced the PCR product yield of the constitutive splicing isoform (Fig. 2BIII vs $B I I)$ and the contamination of the alternative splicing isoform by the constitutive splicing isoform. This approach allowed us to isolate and sequence the alternative splicing isoform (Fig. 2BIII,IV). Importantly, when the pure constitutive splicing isoform was used as the template, primers $1 ' / 2$ ' did not generate the PCR product of the alternative splicing isoform (data not shown), which suggests that primer $2^{\prime}$ cannot loop out the $5^{\prime}$-terminal nine bases in exon 14 of Tmc1 to artificially generate the PCR product of the alternative splicing isoform.

\section{Differential expression of TMC1 and LHFPL5 isoforms}

Splicing isoforms of proteins are widely suggested to display tissue-specific or cell-specific expression patterns. To test whether TMC1 and LHFPL5 splicing isoforms exhibit tissue-specific expression, we performed 
A

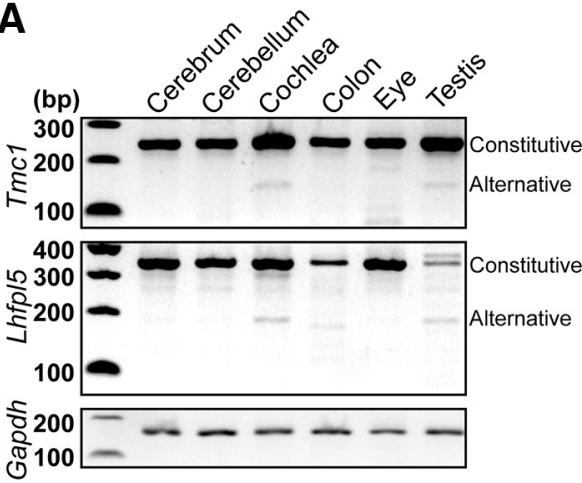

C
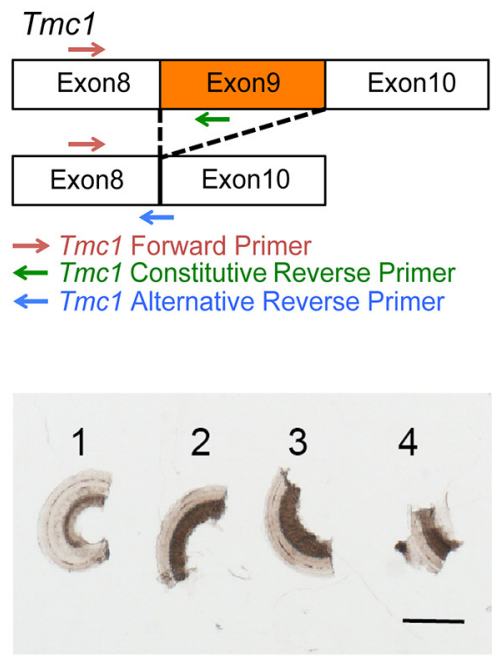

B

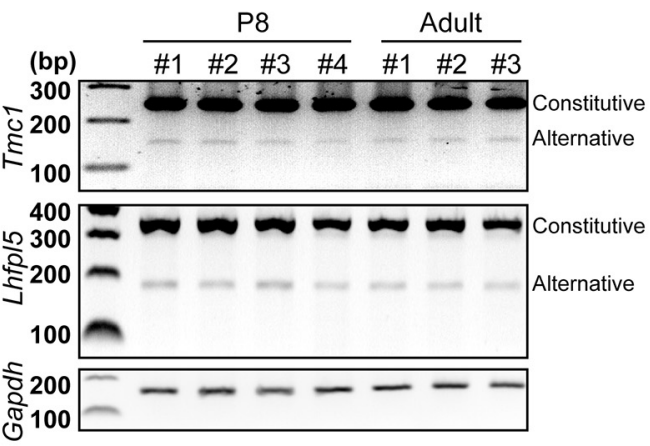

Lhfp/5
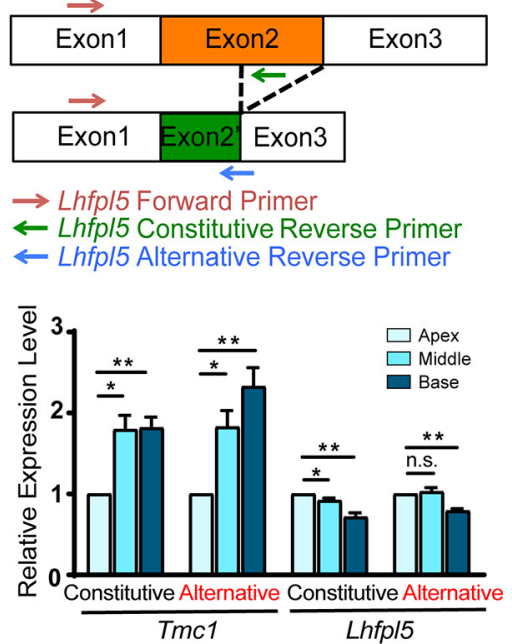

Figure 3. Differential expression of TMC1 and LHFPL5 isoforms. Total RNA was extracted from different tissues from P31-P33 mice $(\boldsymbol{A})$ or cochleae from P8 and adult (P32-P40) mice (B), and mRNA was reverse-transcribed into cDNA by using oligo-dT primers. Approximately $30 \mathrm{ng}$ of starting cDNA from each tissue was used as the PCR template, and final amplified products were separated on agarose gels. Bands representing constitutive and alternative splicing isoforms are labeled. GAPDH, loading control. C, upper panel, Schematic of primers used in QRT-PCR of constitutive and alternative splicing isoforms. Lower panel, left, Organ of Corti from P6 mice was dissected into four segments. Segments 1-3: apical, middle, and basal parts of organ of Corti. Segment 4 was discarded because it was frequently damaged during dissection. Samples were fixed to flatten the coiled segments and thereby improve their imaging. Scale bar: $100 \mu \mathrm{m}$. Lower panel, right, Results of qRT-PCR. $N=5$ independent biological replicates. From left to right: for Tmc1, ${ }^{\star} p=0.010,{ }^{* *} p=0.003,{ }^{*} p=0.015,{ }^{* *} p=0.005$; for Lhfp/5, ${ }^{*} p=0.049,{ }^{* *} p=0.005$, n.s., not significant, ${ }^{* *} p=0.002$.

semiquantitative RT-PCR to examine the expression of the isoforms in various tissues (Fig. 3). In agreement with previous work suggesting the presence of TMC1 mRNA in six tested tissues (Keresztes et al., 2003), the constitutive splicing TMC1 isoform was amplified from the cDNAs generated from these tissues (Fig. $3 A$ ). Furthermore, these tissues were also found to contain LHFPL5 mRNA (Fig. $3 A$ ). Interestingly, a lower band representing the alternatively spliced isoform of TMC1 and LHFPL5 was detected only in the cochlea and testis. These results suggest that TMC1 and LHFPL5 isoforms potentially perform tissue-specific functions.

We next examined the developmental profile of TMC1 and LHFPL5 splicing isoforms in the cochlea. The cDNAs generated from four mouse pups (at P8) and three adult mice (two at P32 and one at P40) were amplified using primers for TMC1 and LHFPL5 isoforms.
Our results showed that the TMC1 and LHFPL5 alternatively spliced isoforms, similar to the constitutively spliced isoforms, appeared at P8 and remained detectable at adulthood (Fig. 3B), this suggests that the TMC1 and LHFPL5 spliced isoforms could play critical roles throughout life in hair cells.

We also assessed the tonotopic gradient of the constitutive and alternative splicing isoforms of TMC1 and LHFPL5. Considering that the tonotopic gradient might be too small to allow detection by using semiquantitative methods, we performed the comparatively more sensitive qRT-PCR assay with primers specifically targeting the constitutive and alternative splicing isoforms. Our results suggest the existence of an upward apicobasal gradient for the constitutive splicing TMC1 isoform in the organ of Corti (Fig. $3 C$ ), this is consistent with the previously reported tonotopic gradient of TMC1 protein in hair bundles 

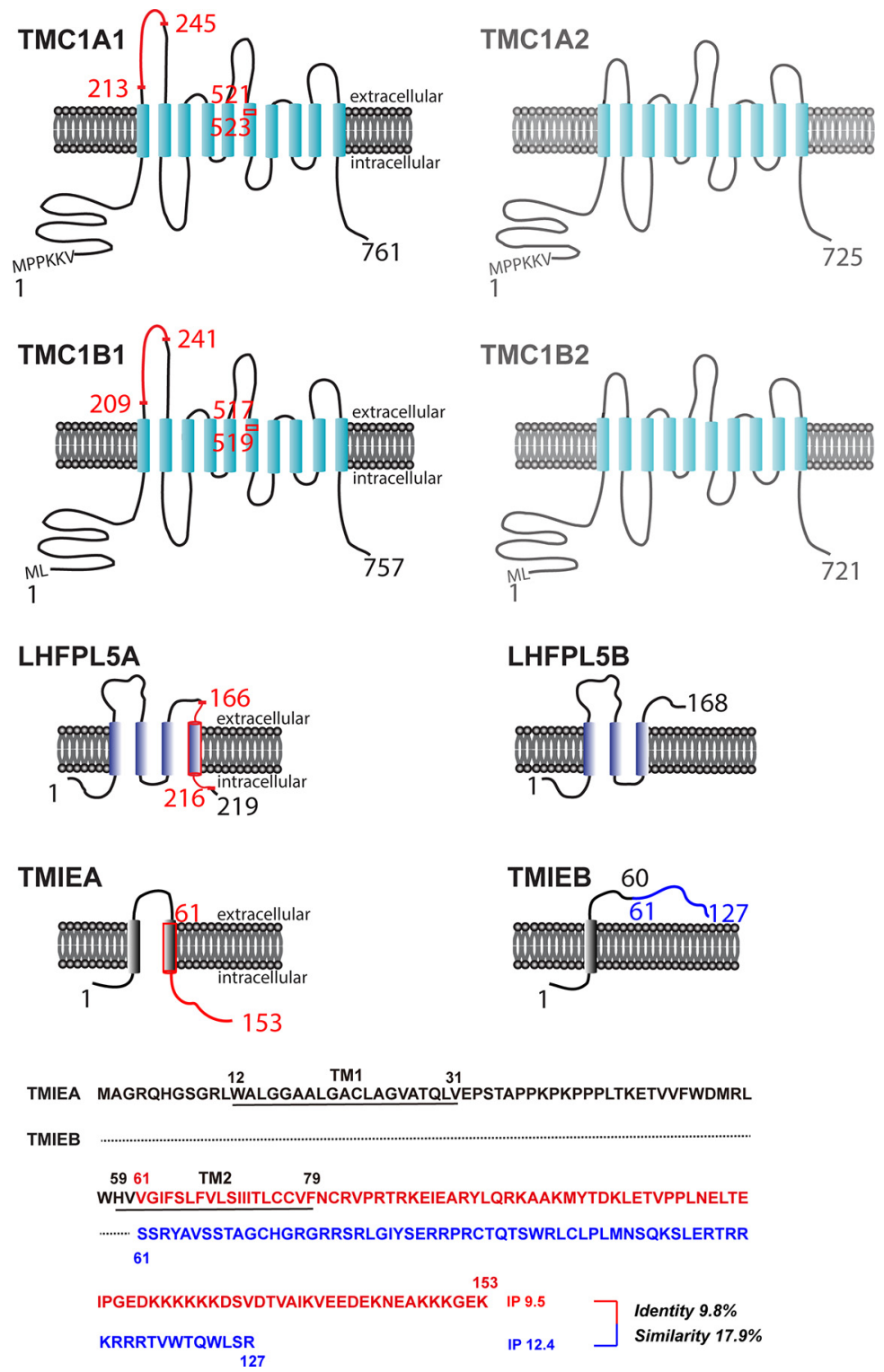

Figure 4. Schematic depiction of splicing variants of TMC1, LHFPL5, and TMIE. Transmembrane domains are shown as columns, and intracellular/extracellular domains are indicated by lines. For each protein, alternatively spliced-out regions and their starting or ending amino acids are shown in red. For TMIEB, the $C$ terminus generated by alternative splicing and reading-frame shift is highlighted in blue. Bottom panel, C-terminal sequences of TMIEB (blue) and TMIEA (red); dotted line, amino acids identical to that in TMIEA; IP, isoelectric point.

(Beurg et al., 2018). By contrast, the constitutive splicing LHFPL5 isoform displayed a modest downward apicobasal gradient (Fig. 3C). Moreover, for both TMC1 and LHFPL5, the tonotopic gradient of the alternative splicing isoforms was the same as that of the constitutive splicing isoforms (Fig. 3C), which suggests that an unbiased splicing machinery operates throughout the organ of Corti.

\section{Schematic protein topology of mouse TMC1, LHFPL5, and TMIE isoforms}

To help visualize the potential functional consequences of the alternative splicing events reported here, we schematically depicted the topologies of the identified
TMC1, LHFPL5, and TMIE isoforms (Fig. 4). The topology of TMC1 is based on two recent homology models (Ballesteros et al., 2018; Pan et al., 2018) rather than on an early model based on hydropathy and epitope accessibility (Labay et al., 2010), the topologies of LHFPL5 and TMIE are based on a 3D structure (Ge et al., 2018) and a predicted model (Zhao et al., 2014), respectively.

TMC1A (NCBI accession: XP_036017315) and TMC1B (NCBI Accession: NP_083229), the TMC1 isoforms starting at exon 1 (and skipping exon 2) and exon 2, respectively, differ only in five residues at the extreme $\mathrm{N}$ terminus: MPPKKV in TMC1A and ML in TMC1B (Fig. 4). We designated the full-length, constitutive splicing 
isoforms of TMC1A and TMC1B as TMC1A1 and TMC1B1 and the alternative splicing isoforms as TMC1A2 and TMC1B2. Both TMC1A1 and TMC1B1 might undergo both of the newly identified alternative splicing events: (1) exon 9 skipping, which deletes amino acids 213-245 (TMC1A) or amino acids 209-241 (TMC1B) in the first extracellular loop; and (2) alternative splicing at exon 14, which deletes amino acids 521-523 (TMC1A) or amino acids 517-519 (TMC1B) in the sixth transmembrane domain. Although these splicing events might occur separately in one transcript, for simplicity, we have tentatively designated TMC1A2 and TMC1B2 as including both the alternative splicing events. The spliced-out segments in TMC1A or TMC1B are highlighted by solid red lines in Figure 4.

LHFPL5 features two isoforms: (1) LHFPL5A (NCBI Accession: NP_080847), full-length, constitutive splicing isoform; and (2) LHFPL5B, in which alternative splicing occurs at exon 2 and results in the deletion of amino acids 166-216, a region containing part of the second extracellular loop, the fourth transmembrane domain, and almost the entire $\mathrm{C}$ terminus (Fig. 4).

TMIE is also expressed as two isoforms: (1) TMIEA (NCBI Accession: NP_666372), full-length, constitutive splicing isoform; and (2) TMIEB, in which alternative splicing occurs at exon four and leads to a reading-frame shift starting at amino acid 61 in the extracellular domain, thus producing a final protein product containing one transmembrane domain and a total of 127 amino acids (Fig. 4).

\section{Discussion}

Among the four known proteins assembled in the MT complex, namely PCDH15, TMC1, LHFPL5, and TMIE, $\mathrm{PCDH} 15$ has been reported to feature three isoforms: CD1, CD2, and CD3 (Ahmed et al., 2001; Webb et al., 2011; Pepermans et al., 2014). However, little is known regarding the alternative splicing of the other 3 MT-complex components. In the case of TMC1, previous studies have suggested the existence of two isoforms with alternative start codons in mice (TMC1A and TMC1B; Fig. 4; Kawashima et al., 2011). Here, we report two previously unidentified alternative splicing events in Tmc1, exon 9 skipping and alternative $3^{\prime}$ splicing in exon 14 (Fig. 4), and we also identify alternative splicing events in $L$ hfp/5 and Tmie (Fig. 4). Notably, the alternative splicing sites in the three genes are highly conserved in mice and humans, which suggests that the alternative splicing events are highly likely to occur in humans as well.

Intriguingly, both Tmc1 and Lhfp/5 display a tissue-specific splicing pattern in the cochlea and testis. Because TMC1 and LHFPL5 play critical roles in hair-cell function, alternative splicing might be functionally important in the case of both proteins (see below). By contrast, the roles of TMC1 and LHFPL5 in the testis have not been closely examined, although knocking TMC1 or LHFPL5 does not appear to affect fertility (Longo-Guess et al., 2007; Kurima et al., 2015). The functions in the testis of these two proteins (of both their constitutive and alternative splicing isoforms) warrant further investigation.
Our data indicate an upward apicobasal gradient for the constitutively spliced TMC1 mRNA in the organ of Corti (Fig. $3 C$ ), this roughly agrees with the tonotopic gradient of TMC1 protein expression in hair bundles (Beurg et al., 2018). By contrast, the constitutively spliced LHFPL5 mRNA displayed a modest downward apicobasal gradient (Fig. 3C); however, further investigation is required to determine whether and how this gradient corresponds to global and hair-bundle LHFPL5 protein expression in hair cells. Moreover, the tonotopic gradient of the alternative splicing isoforms of both genes was the same as that of the constitutive splicing isoforms (Fig. $3 \mathrm{C}$ ), and the lack of a tonotopic gradient in the splicing ratio of the two genes suggests the existence of an unbiased splicing machinery across the entire organ of Corti.

What are the potential functions of the alternative splicing isoforms of the MT-complex proteins? The three splice isoforms of PCDH15, CD1, CD2, and CD3, differ only in their cytoplasmic domains, as a result of exclusion and inclusion of exons through alternative splicing (Webb et al., 2011; Pepermans et al., 2014), and whereas the isoforms are functionally redundant at an early developmental stage in mice, CD2 becomes critical in mature hair cells (Pepermans et al., 2014). Conversely, the difference in the cytoplasmic domain of the isoforms regulates the interaction between PCDH15 and TMIE (Zhao et al., 2014).

The two isoforms of TMC1 featuring alternative translation initiation sites (TMC1A and TMC1B) differ only within the first 5 amino acid residues at the $\mathrm{N}$ terminus (Fig. 4), and although the functional difference between the two isoforms remains unclear, it is expected to be minor (Yamaguchi et al., 2020). Both isoforms can rescue the transducer current in TMC1-knock-out mice (Kawashima et al., 2011; Nist-Lund et al., 2019; Cunningham et al., 2020). Exon 9 skipping deletes amino acids 213-245 in TMC1A (amino acids 209-241 in TMC1B) in the first putative extracellular loop, and this 33 -aa segment contains an alternative $\mathrm{N}$-linked glycosylation site $\mathrm{NXG}, \mathrm{N}_{237} \mathrm{FG}$, in TMC1A (Lowenthal et al., 2016). Because N-glycosylation is involved in protein folding and function, splicing out this segment might affect TMC1 folding and function. Furthermore, alternative splicing at exon 14 deletes amino acids 521-523 in TMC1A (amino acids 517-519 in TMC1B) in the sixth transmembrane domain (Fig. 4), this could affect hearing by disrupting TMC1 channel function: the spliced-out three residues, V521GQ, appear to form part of the channel pore in the published homology models (Ballesteros et al., 2018; Pan et al., 2018).

Interestingly, the GluR6a subunit of ionotropic glutamate receptors has been shown to promote cell-surface expression of its splicing variant GluR6b and several GluR5 splicing variants (Jaskotski et al., 2004). Thus, it will be of interest to investigate how the alternative splicing isoforms of TMC1 modulate the trafficking of the constitutive splicing isoform of TMC1.

Alternative splicing in exon 2 of LHFPL5 generates the LHFPL5B isoform; the splicing deletes amino acids 166216 , a region that includes part of the second extracellular loop, the fourth transmembrane domain, and almost the 
entire $C$ terminus (Fig. 4). Notably, LHFPL5B by itself failed to support any hearing function: profound deafness was recorded in homozygous $L h f p / 5^{h s c y-2 J}$ mice $\left(L h f p / 5^{B / B}\right.$ mice expressing the LHFPL5B isoform; Longo-Guess et al., 2007). Moreover, the LHFPL5B gene (resulting from the hscy-2J mutation) was suggested to be recessive because heterozygous Lhfp/5 $5^{h s c y-2 J}$ mice showed normal hearing. However, this notion remains to be confirmed because it did not account for the finding that the wild-type allele in the heterozygous mice produces not only LHFPL5A through canonical splicing, but also LHFPL5B through alternative splicing: in principle, the wild-type allele could produce a sufficient amount of LHFPL5B and thereby mask the dominant (positive or negative) effect of LHFPL5B generated by the mutant allele (hscy-2J). Therefore, how LHFPL5B affects LHFPL5A protein function remains unelucidated.

Alternative splicing in exon four of TMIE, which generates TMIEB, causes a reading-frame shift starting after amino acid 60 in the extracellular domain and produces a final protein product that contains one transmembrane domain and is 127 aa long (Fig. 4). The $\mathrm{C}$ terminus (amino acids 61-127) in TMIEB shares little homology with the TMIEA C terminus (amino acids 61-153; 9.8\% identify and $17.9 \%$ similarity) and does not appear to contain any transmembrane helices according to TMpred (data not shown), this algorithm is used for statistical analysis of TMbase, which stores naturally occurring transmembrane proteins (Hofmann and Stoffel, 1993). Because the C-terminal half of TMIEA has been found to be critical for binding to TMC1, PIP2, PCDH15, and LHFPL5 (Zhao et al., 2014; Cunningham et al., 2020), TMIEB might potentially not support any normal hearing function. TMIEB could act as a dominant-negative regulator of TMIEA function if TMIEB, particularly its N-terminal 60 aa shared with TMIEA, still binds to a known or unknown component of the MT complex. Whether the N terminus of TMIE binds to any MT-complex component is currently unknown, although the first 28 aa of TMIE appear to mediate the MT-channel response to mechanical cues (Cunningham et al., 2020). Interestingly, the $C$ terminus of TMIEB, similar to that of TMIEA, is also highly positively charged (Fig. 4) and might interact with PIP2 (Cunningham et al., 2020).

In summary, we have identified previously unreported splicing variants of TMC1, LHFPL5, and TMIE, the pivotal molecules forming the hair-cell MT machinery. Our findings reveal the potential complexity of the MT-complex composition and provide valuable guidance for future research on the function, regulation, and trafficking of TMC1, LHFPL5, and TMIE. Furthermore, our study could help direct the clinical diagnosis of hearing loss related to aberrant splicing of TMC1, LHFPL5, and TMIE. The key question to address next is the physiological function of the alternative splicing isoforms of these proteins critical for normal hearing.

\section{References}

Ahmed ZM, Riazuddin S, Bernstein SL, Ahmed Z, Khan S, Griffith AJ, Morell RJ, Friedman TB, Riazuddin S, Wilcox ER (2001) Mutations of the protocadherin gene PCDH15 cause usher syndrome type 1F. Am J Hum Genet 69:25-34.
Ahmed ZM, Goodyear R, Saima R, Lagziel A, Legan PK, Behra M, Burgess SM, Lilley KS, Wilcox ER, Sheikh R, Griffith AJ, Frolenkov GI, Belyantseva IA, Richardson GP, Friedman TB (2006) The tiplink antigen, a protein associated with the transduction complex of sensory hair cells, is protocadherin-15. J Neurosci 26:7022-7034.

Ballesteros A, Fenollar-Ferrer C, Swartz KJ (2018) Structural relationship between the putative hair cell mechanotransduction channel TMC1 and TMEM16 proteins. Elife 7:e38433.

Baralle FE, Giudice J (2017) Alternative splicing as a regulator of development and tissue identity. Nat Rev Mol Cell Biol 18:437-451.

Bartsch TF, Hengel FE, Oswald A, Dionne G, Chipendo IV, Mangat SS, El Shatanofy M, Shapiro L, Müller U, Hudspeth AJ (2019) Elasticity of individual protocadherin 15 molecules implicates tip links as the gating springs for hearing. Proc Natl Acad Sci USA 116:11048-11056.

Beurg M, Xiong W, Zhao B, Müller U, Fettiplace R (2015) Subunit determination of the conductance of hair-cell mechanotransducer channels. Proc Natl Acad Sci USA 112:1589-1594.

Beurg M, Cui R, Goldring AC, Ebrahim S, Fettiplace R, Kachar B (2018) Variable number of TMC1-dependent mechanotransducer channels underlie tonotopic conductance gradients in the cochlea. Nat Commun 9:2185.

Cunningham CL, Qiu X, Wu Z, Zhao B, Peng G, Kim YH, Lauer A, Müller U (2020) TMIE defines pore and gating properties of the mechanotransduction channel of mammalian cochlear hair cells. Neuron 107:126-143.e8.

Ge J, Elferich J, Goehring A, Zhao H, Schuck P, Gouaux E (2018) Structure of mouse protocadherin 15 of the stereocilia tip link in complex with LHFPL5. Elife 7:e38770.

Hofmann K, Stoffel W (1993) Konferenz der Gesellschaft für Biologische Chemie Studiengruppe Membranstruktur und -Transport Membranforum Frankfurt. Biol Chem Hoppe Seyler 374: 143-170.

Jaskotski F, Coussen F, Nagarajan N, Normand E, Rosenmund C, Mulle C (2004) Subunit composition and alternative splicing regulate membrane delivery of kainate receptors. J Neurosci 24:25062515.

Kawashima Y, Géléoc GSG, Kurima K, Labay V, Lelli A, Asai Y, Makishima T, Wu DK, Della Santina CC, Holt JR, Griffith AJ (2011) Mechanotransduction in mouse inner ear hair cells requires transmembrane channel-like genes. J Clin Invest 121:4796-4809.

Keresztes G, Mutai H, Heller S (2003) TMC and EVER genes belong to a larger novel family, the TMC gene family encoding transmembrane proteins. BMC Genomics 4:24.

Kim KX, Fettiplace R (2013) Developmental changes in the cochlear hair cell mechanotransducer channel and their regulation by transmembrane channel-like proteins. J Gen Physiol 141:141-148.

Kurima K, Peters LM, Yang Y, Riazuddin S, Ahmed ZM, Naz S, Arnaud D, Drury S, Mo J, Makishima T, Ghosh M, Menon PSN, Deshmukh D, Oddoux C, Ostrer H, Khan S, Riazuddin S, Deininger PL, Hampton LL, Sullivan SL, et al. (2002) Dominant and recessive deafness caused by mutations of a novel gene, TMC1, required for cochlear hair-cell function. Nat Genet 30:277-284.

Kurima K, Ebrahim S, Pan B, Sedlacek M, Sengupta P, Millis BA, Cui R, Nakanishi H, Fujikawa T, Kawashima Y, Choi BY, Monahan K, Holt JR, Griffith AJ, Kachar B (2015) TMC1 and TMC2 localize at the site of mechanotransduction in mammalian inner ear hair cell stereocilia. Cell Rep 12:1606-1617.

Kwok S, Kellogg DE, Mckinney N, Spasic D, Goda L, Levenson C, Sninsky JJ (1990) Effects of primer-template mismatches on the polymerase chain reaction: human immunodeficiency virus type 1 model studies. Nucleic Acids Res 18:999-1005.

Labay V, Weichert RM, Makishima T, Griffith AJ (2010) Topology of transmembrane channel-like gene 1 protein. Biochemistry 49:8592-8598.

Li Y, Liu H, Giffen KP, Chen L, Beisel KW, He DZZ (2018) Transcriptomes of cochlear inner and outer hair cells from adult mice. Sci Data 5:180199.

Liu Y, Gonzàlez-Porta M, Santos S, Brazma A, Marioni JC, Aebersold R, Venkitaraman AR, Wickramasinghe VO (2017) 
Impact of alternative splicing on the human proteome. Cell Rep 20:1229-1241.

Longo-Guess CM, Gagnon LH, Cook SA, Wu J, Zheng QY, Johnson KR (2005) A missense mutation in the previously undescribed gene Tmhs underlies deafness in hurry-scurry (hscy) mice. Proc Natl Acad Sci USA 102:7894-7899.

Longo-Guess CM, Gagnon LH, Fritzsch B, Johnson KR (2007) Targeted knockout and lacZ reporter expression of the mouse Tmhs deafness gene and characterization of the hscy-2J mutation. Mamm Genome 18:646-656.

Lowenthal MS, Davis KS, Formolo T, Kilpatrick LE, Phinney KW (2016) Identification of novel N-glycosylation sites at noncanonical protein consensus motifs. J Proteome Res 15:2087-2101.

Naz S, Giguere CM, Kohrman DC, Mitchem KL, Saima R, Morell RJ, Ramesh A, Srisailpathy S, Deshmukh D, Sheikh R, Griffith AJ, Friedman TB, Smith RJH, Wilcox ER (2002) Mutations in a novel gene, TMIE, are associated with hearing loss linked to the DFNB6 locus. Am J Hum Genet 71:632-636.

Nist-Lund CA, Pan B, Patterson A, Asai Y, Chen T, Zhou W, Zhu H, Romero S, Resnik J, Polley DB, Géléoc GS, Holt JR (2019) Improved TMC1 gene therapy restores hearing and balance in mice with genetic inner ear disorders. Nat Commun 10:236.

Pan B, Géléoc GS, Asai Y, Horwitz GC, Kurima K, Ishikawa K, Kawashima Y, Griffith AJ, Holt JR (2013) TMC1 and TMC2 are components of the mechanotransduction channel in hair cells of the mammalian inner ear. Neuron 79:504-515.

Pan B, Akyuz N, Liu XP, Asai Y, Nist-Lund C, Kurima K, Derfler BH, György B, Limapichat W, Walujkar S, Wimalasena LN, Sotomayor M, Corey DP, Holt JR (2018) TMC1 forms the pore of mechanosensory transduction channels in vertebrate inner ear hair cells. Neuron 99:736-753.e6.

Pan Q, Shai O, Lee LJ, Frey BJ, Blencowe BJ (2008) Deep surveying of alternative splicing complexity in the human transcriptome by high-throughput sequencing. Nat Genet 40:1413-1415.

Pepermans E, Michel V, Goodyear R, Bonnet C, Abdi S, Dupont T, Gherbi S, Holder M, Makrelouf M, Hardelin J, Marlin S, Zenati A, Richardson G, Avan P, Bahloul A, Petit C (2014) The CD 2 isoform of protocadherin-15 is an essential component of the tip-link complex in mature auditory hair cells. EMBO Mol Med 6:984-992.

Pertea M, Kim D, Pertea GM, Leek JT, Salzberg SL (2016) Transcript-level expression analysis of RNA-seq experiments with HISAT, StringTie and Ballgown. Nat Protoc 11:1650-1667.

Rinné S, Renigunta V, Schlichthörl G, Zuzarte M, Bittner S, Meuth SG, Decher N, Daut J, Preisig-Müller R (2014) A splice variant of the two-pore domain potassium channel TREK-1 with only one pore domain reduces the surface expression of full-length TREK-1 channels. Pflugers Arch Eur 466:1559-1570.

Stadhouders R, Pas SD, Anber J, Voermans J, Mes THM, Schutten M (2010) The effect of primer-template mismatches on the detection and quantification of nucleic acids using the $5^{\prime}$ nuclease assay. J Mol Diagnostics 12:109-117.

Szczot M, Pogorzala LA, Solinski HJ, Young L, Yee P, Le Pichon CE, Chesler AT, Hoon MA (2017) Cell-type-specific splicing of Piezo2 regulates mechanotransduction. Cell Rep 21:2760-2771.

Webb SW, Grillet N, Andrade LR, Xiong W, Swarthout L, Santina CCD, Kachar B, Müller U (2011) Regulation of PCDH15 function in mechanosensory hair cells by alternative splicing of the cytoplasmic domain. Development 138:1607-1617.

Xiong W, Grillet N, Elledge HM, Wagner TFJ, Zhao B, Johnson KR, Kazmierczak P, Müller U (2012) TMHS is an integral component of the mechanotransduction machinery of cochlear hair cells. Cell 151:1283-1295.

Yamaguchi S, Hamamura M, Otsuguro KI (2020) A mechanosensitive channel, mouse transmembrane channel-like protein 1 (Mtmc1) is translated from a splice variant $m T m c 1$ ex 1 but not from the other variant $m$ Tmc1ex2. Int J Mol Sci 21:6465.

Yu X, Zhao Q, Li X, Chen Y, Tian Y, Liu S, Xiong W, Huang P (2020) Deafness mutation D572N of TMC1 destabilizes TMC1 expression by disrupting LHFPL5 binding. Proc Natl Acad Sci USA 117:29894-29903.

Zhao B, Wu Z, Grillet N, Yan L, Xiong W, Harkins-Perry S, Mü LU (2014) TMIE is an essential component of the mechanotransduction machinery of cochlear hair cells. Neuron 84:954-967. 